\title{
A PROPOS DU RASSASIEMENT DE LA VACHE LAITIËRE
}

PAR

\author{
André M. LEROY
}

Professeur de Zootechnie à l'Institut National Agronomique

Le travail que vient de publier M. A. Vorsin, avec l'aide des“données]de Fissmer, possède un très grand intérêt, parce qu'il attire l'attention sur les sources éventuelles de la déperdition d'énergie qu'entraîne la consommation des aliments par les ruminants. Mais il est regrettable que les calculs auxquels il aboutit soient en contradiction formelle avec l'état actuel de nos connaissances concernant la physiologie de la nutrition.

La critique de l'utilité des tables d'alimentation, à laquelle l'auteur précité a réservé son préambule, ne nous paraît pas fondée. Notre expérience de l'alimentation de la vache, acquise en une quarantaine d'années, ne nous conduit nullement à un pareil excès de pessimisme, et l'emploi systématique des normes scandinaves et françaises rend incontestablement dans la pratique d'excellents services. En particulier, le type de ration que paraît condamner M. Vorsin est presqu'exactement celui que nous avons choisi pour le troupeau laitier du domaine expérimental de Jouy-en-Josas, au cours de 1'hiver I9.50I95I, ce qui nous a permis d'obtenir une production moyenne par tête de I $8 \mathrm{~kg}$ de lait à $4 \%$ de matière grasse en octobre, laquelle a diminué lentement et d'une manière conforme à la théorie jusqu'à $15 \mathrm{~kg}$ en mai, au moment de la mise à l'herbe. Les excellents effets de cette alimentation strictement contrôlée par la pesée de tous ses éléments avec évaluation des refus nous ont été prouvés par l'absence de l'augmentation de cette production après la mise en pâture, laquelle est ordinairement la règle dans les troupeaux insuffisamment nourris dans le cours de l'hiver.

Ajoutons également qu'un plan de rationnement du même type avait été utilisé par nous avec le plus grand succès, au cours d'expériences poursuivies en Seine-Inférieure, chez M. MonvILLE, pendant l'hiver I949-I950, sous la direction de notre collaborateur S. ZELTER.

Enfin, toutes les étables de Seine-et-Marne, soumises au contrôle laitier, 
sont actuellement peuplées d'animaux alimentés d'après la méthode exposée dans maints ouvrages, au moyen d'une ration de base de pulpe ou de betteraves et d'ensilage, avec 4 à $5 \mathrm{~kg}$ de foin et 3 à $4 \mathrm{~kg}$ de menue-paille, complétée par un mélange concentré équilibré en matières azotées, distribué à raison de $450 \mathrm{~g}$, environ par $\mathrm{kg}$ de lait produit en plus d'une base de production de 8 litres. Si nous avions pu étudier avec M. Vorsin la variation de production constatée par lui en passant de l'ensilage de luzerne à l'ensilage de feuilles et collets, nous ne doutons pas que nous aurions trouvé la raison de ce qui peut apparaître $a$ priori comme une anomalie, mais qui pourrait peut-être provenir d'une différence de qualité entre les deux ensilages. L'appétence pour une nourriture donnée est un facteur d'une grande importance pratique le plus souvent négligée par les théoriciens, mais que M. Vorsin met fort bien en évidence dans son propre rapport, car il est certain que les vaches n'acceptent pas volontiers des doses d'ensilage de luzerne supérieures à $25 \mathrm{~kg}$, surtout lorsque le fourrage a été coupé à la fíraison, ce qui est le cas général.

Tout en reconnaissant l'intérêt de la théorie du ballast, imaginée par LEHMANN, que notre excellent collègue et ami CRASEMANN s'efforce de faire connaître, nous pensons qu'elle exprime d'une manière compliquée ce qui peut se mesurer d'une manière plus simple par le rapport de la valeur énergétique nette de l'aliment à la quantité de matière sèche correspondante.

Dire que le coefficient d'encombrement d'une ration d'herbe contenant $27,5 \%$ de cellulose par rapport à la matière sèche est de $\mathbf{I}, 4$, cela signifie que pour I $\mathrm{kg}$ de matière sèche de l'herbe ingérée la valeur fourragère correspondante est de 0,77 unité fourragère, ou de 0,54 unité-amidon-engraissement selon KELLNER. Dans ce kg de matière sèche, dont le coefficient.de digestibilité est de 0,70 , la quantité de "ballast " (matériaux indigestibles) est de $300 \mathrm{~g}$. Mais pourquoi attribuer à la matière indigestible seule le pouvoir de lester le tube digestif ? Ce qui correspond à l'effort musculaire de transit du bol alimentaire depuis la bouche jusqu'à l'anus, c'est évidemment la moyenne :

\section{$\frac{\text { Poids de la matière sèche ingérée }+ \text { Poids du ballast }}{2}$}

et non pas le "ballast " seul. En fait, la théorie du coefficient d'encombrement est plus souple que celle du ballast, car si nous disons qu'une bonne laitière (à I5-20 kg de lait) doit recevoir une alimentation dont le coefficient d'encombrement $\frac{\mathrm{MS}}{\mathrm{U}}$ se rapproche de celui de l'herbe - I,2 - I,4 - nous comprenons aisément qu'à production laitière égale, une bonne vache normande de $700 \mathrm{~kg}$ peut fort bien tolérer une ration dont le coefficient d'encombrement s'élève à la rigueur jusqu'à $I, 6$, tandis qu'une ration ayant le coefficient théorique $I, 4$ peut se montrer trop encombrante pour une bretonne de $350 \mathrm{~kg}$ dont 1'appareil digestif est plus petit.

Au sujet du coefficient d'encombrement, nous nous permettons d'attirer 
l'attention du lecteur sur la communication publiée sous notre signature dans le Bulletin technique d'Information du Ministère de l'Agriculture, $n^{0} 4^{6}$, janvier I950. Sous l'impression des discussions auxquelles nous avions participé, pendant les journées d'étude de l'alimentation animale, organisées en I949 à Zurich par la F. A. O., nous avons conçu ce travail en pensant principalement aux conséquences pratiques qui peuvent en découler.

Dans les circonstances ordinaires de sa vie, une vache laitière doit pouvoir consommer chaque jour, en une durée totale de 6 à 7 heures, une masse de matière sèche en relation avec sa masse corporelle, qui, pour une vache de $600 \mathrm{~kg}$ douée d'un appétit normal, peut atteindre de $\mathrm{I} 3$ à $\mathrm{I} 5 \mathrm{~kg}$. Plus cette quantité de matière sèche contiendra d'éléments digestibles, plus sa valeur énergétique, exprimée en unités fourragères, sera considérable. Ainsi, pour une masse d'herbe verte d'un pâturage ordinaire de 65 à $7 \mathrm{o} \mathrm{kg}$, qui compose la ration consommée sur place par une vache dont la production moyenne est de $15 \mathrm{~kg}$ d'un lait à $4 \%$ de matière grasse, la matière sèche consommée est de $13.5 \mathrm{~kg}$, tandis que la valeur fourragère correspondante est d'environ to unités. Le coefficient d'encombrement correspondant $\frac{\mathrm{MS}}{\mathrm{U}}$ est donc de I,35.

On trouverait de même, en consultant une table d'alimentation, que la ration composée de foin ordinaire contenant la même quantité de matière sèche $-13,5 \mathrm{~kg}$ - ne possède qu'une valeur fourragère de 7,6 unités. Or, la ration nécessaire à l'entretien d'une vache de $600 \mathrm{~kg}$ est de 4,4 unités, de telle sorte que la part de l'énergie des aliments disponible pour la production est de 3,2 unités $(4,4+3,2=7,6)$. Sachant que le besoin, pour la production d'un $\mathrm{kg}$ de lait est de 0,38 unité, on voit que la production laitière de base, c'est-à-dire celle qu'il est possible d'obtenir sans apport supplémentaire d'aliments concentrés, est de $8,3 \mathrm{~kg}$.

Le tableau I (page 5o) donne, pour des rations de base contenant la même quantité de matière sèche - I3,5 kg - les valeurs correspondant à l'apport énergétique, à la production laitière obtenue sans concentrés, et au coefficient d'encombrement $\frac{M S}{U}$. L'examen de ce tableau montre l'existence d'une relation évidente entre la production laitière de base et la valeur de $\frac{\mathrm{MS}}{\mathrm{U}}$.

$\mathrm{Si}$, à l'aide de deux axes de coordonnées, nous portons sur l'axe des abscisses les valeurs de $\frac{M S}{U}$, et, sur celui des ordonnées, les valeurs correspondantes de ce que nous appelons la production laitière de base - production maximum qui peut être obtenue sans addition d'aliments concentrés - nous constatons que les points qui représentent les Io couples de données sont disposés suivant une courbe régullière qui fait penser à un arc d'hyperbole.

I1 est facile de démontrer que cette courbe est bien un arc d'hyperbole de formule $y=\frac{a}{x}+b$, à l'aide du raisonnement ci-après : 


\section{TABLEAU I}

Productions laitières de base correspondant à des rations contenant la même quantité de matière sèche, $13,5 \mathrm{~kg}$, mais qui possèdent des coefficients d'encombrement progressivement croissants.

\begin{tabular}{|c|c|c|c|c|c|c|}
\hline Nos & Composition de la ration & Kilos & $\begin{array}{l}\text { Valeur } \\
\text { fourragère } \\
\text { totale } \\
\text { en u. f. }\end{array}$ & $\begin{array}{l}\text { Val. fourr. } \\
\text { dispon. } p^{r} \\
\text { la produc- } \\
\text { tion en } u . \Lambda .\end{array}$ & $\begin{array}{l}\text { Quantité } \\
\text { de lait } \\
\text { corresp. } \\
\text { en kilos }\end{array}$ & $\frac{\mathrm{MS}}{\mathrm{L}}$ \\
\hline $\begin{array}{l}\text { I } \\
2 \\
3 \\
4 \\
5 \\
6\end{array}$ & 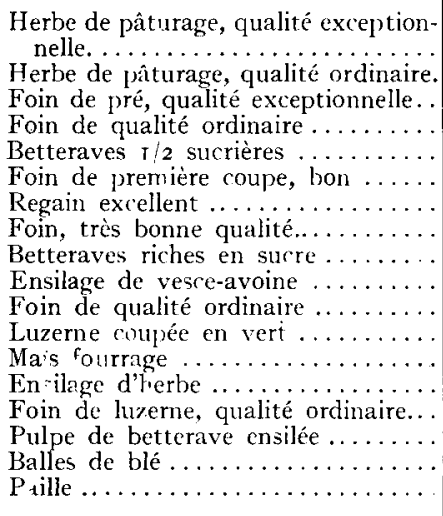 & $\begin{array}{l}62 \\
67,5 \\
16,1 \\
12 \\
26,5 \\
10 \\
5,8 \\
7,5 \\
2,5 \\
27 \\
15,8 \\
40 \\
18 \\
70 \\
5 \\
40 \\
3 \\
3\end{array}$ & $\begin{array}{l}1 \mathrm{I}, 8 \\
10,1 \\
10 \\
9 \\
8,4\end{array}$ & $\begin{array}{l}7,4 \\
5,7 \\
5,6 \\
4,6 \\
4\end{array}$ & $\begin{array}{l}\text { I } 9,5 \\
\text { I } 5 \\
\text { I } 4,7 \\
\text { I } 2, \text { I } \\
\text { I0,5 }\end{array}$ & $\begin{array}{l}\mathrm{I}, \mathrm{I} 3 \\
\mathrm{I}, 34 \\
\mathrm{I}, 35 \\
\mathrm{I}, 50 \\
\mathrm{I}, 61 \\
\\
\mathrm{I}, 73 \\
\mathrm{I}, 78 \\
\mathrm{t}, 30 \\
\mathrm{I}, 93 \\
\\
2,14\end{array}$ \\
\hline
\end{tabular}

Nous savons que si nous désignons par $y$ la quantité de lait fournie par l'animal, exprimée en $\mathrm{kg}$ par jour, le nombre U d'unités fourragères que doit comporter la ration quotidienne est fourni par le calcul suivant :

$$
\text { Io } \quad \mathrm{U}=\mathrm{E}+0,3^{8} y
$$

expression dans laquelle $\mathrm{k}$, est la ration d'entretien, qui est fonction du poids vif de la vache. On sait, en effet, que le besoin énergétique de production par $\mathrm{kg}$ de lait, exprimé en unités fourragères, est de $0,38 \mathrm{U}$.

Si nous posons, $x=\frac{\mathrm{MS}}{\mathrm{U}}$, l'expression $\mathrm{I}^{\circ}$ devient :

$$
2^{0} \quad \mathrm{E}+0,38 y=\frac{\mathrm{MS}}{x} .
$$

En résolvant cette équation par rapport à $y$, nous obtenons :

$$
3^{o} \quad y=\left(\frac{\mathrm{MS}}{x}-\mathrm{E}\right) \frac{\mathrm{I}}{0,3^{8}} \text {. }
$$

D'après notre hypothèse précédente, nous savons que la valeur MS, qui représente la quantité de matière sèche de la ration de base, est de $13,5 \mathrm{~kg}$. D'autre part, nous avons dit que la ration $\mathrm{E}$ d'entretien pour une vache de $600 \mathrm{~kg}$ est de 4,4 unités. En remplaçant MS et E par ces données dans l'équation $3^{\circ}$, il vient finalement :

$$
4^{0} . \quad y=\frac{\mathrm{I}}{0,38}\left(\frac{\mathrm{I} 3,5}{x}-4,4\right),
$$


laquelle est bien de la forme $y=\frac{k}{x}+b$, et peut s'écrire en effectuant les calculs :

$$
5^{\mathrm{o}} \quad y=\frac{35, \mathrm{I}}{x}-\mathrm{II}, 44 \text {. }
$$

Dans cette formule, rappelons-le, $x$ représente le coefficient d'encombrement $\frac{M S}{U}$ de rations de base de composition variable, renfermant toutes I3,5 kg de matière sèche, tandis que $y$ correspond à la production laitière de base.

La courbe définie par cette équation passe d'une manière frappante par les points caractéristiques dont les coordonnées figurent au tableau I, ce qui est une très bonne confirmation de la théorie que nous venons d'exposer.

C'est par la réduction du coefficient d'encombrement des rations de base distribuées à leurs vaches que les éleveurs scandinaves sont parvenus à obtenir autant de lait qu'avant 1940 , avec une consommation de grains et de tourteaux d'importation réduite de $90 \%$ par rapport à celles des années précédant $1 \mathrm{a}$ période de guerre. Conseillés par nous, les Éleveurs de la région Parisienne ont fait leur profit de cet enseignement, et le seuil à partir duquel ils donnent des aliments concentrés à leurs vaches, qui était de $7 \mathrm{~kg}$ auparavant, est maintenant d'environ Io $\mathrm{kg}$, ce qui correspond à une économie journalière de $\mathbf{I , 5} \mathrm{kg}$ d'aliment concentré par vache, avec une production moyenne par tête fortement améliorée.

En reprenant les données originales de FISSMER, nous sommes arrivés à des constatations du plus grand intérêt, dont l'importance nous aurait probablement échappé sans la lecture attentive du travail de M. VoIsin.

La théorie du rassasiement change ainsi d'aspect, ainsi que nous allons le voir :

Les équations précédentes peuvent se mettre sous une autre forme, en remplaçant les valeurs de $y$, qui désignaient des quantités de lait exprimées en $\mathrm{kg}$, par les valeurs fourragères des rations théoriquement capables de couvrir les besoins énergétiques totaux correspondant à ces productions. Les calculs permettant ces remplacements s'effectuent de la manière suivante, pour une vache de $600 \mathrm{~kg}$ donnant un lait à $4 \%$ de matière grasse :

Valeur fourragère pour Io $\mathrm{kg}$ de lait :

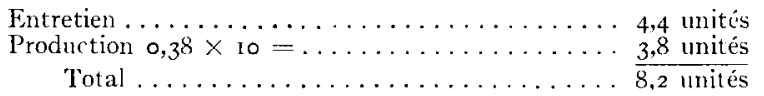

Pour une vache qui consomme $13,5 \mathrm{~kg}$ de matière sèche, la variation de la valeur fourragère de la ration de base, en fonction du coefficient d'encombrement, s'exprime par la même équation, de la forme $y=\frac{k}{x}-a$, que celle qui correspond à la production laitière. La courbe correspondante a été reproduite sur le dessin ci-joint (graphique $n^{\circ} I$ ). 
Mais supposons que l'appétit de la vache pour la ration dé base qu'on lui présente soit augmenté ou diminué. Pour chaque quantité conventionnelle de matière sèche ingérée, I5 kg-I2 $\mathrm{kg}$ - Io,5 $\mathrm{kg}-9 \mathrm{~kg}$, nous pouvons, en reprenant exactement notre raisonnement, construire les courbes correspondante

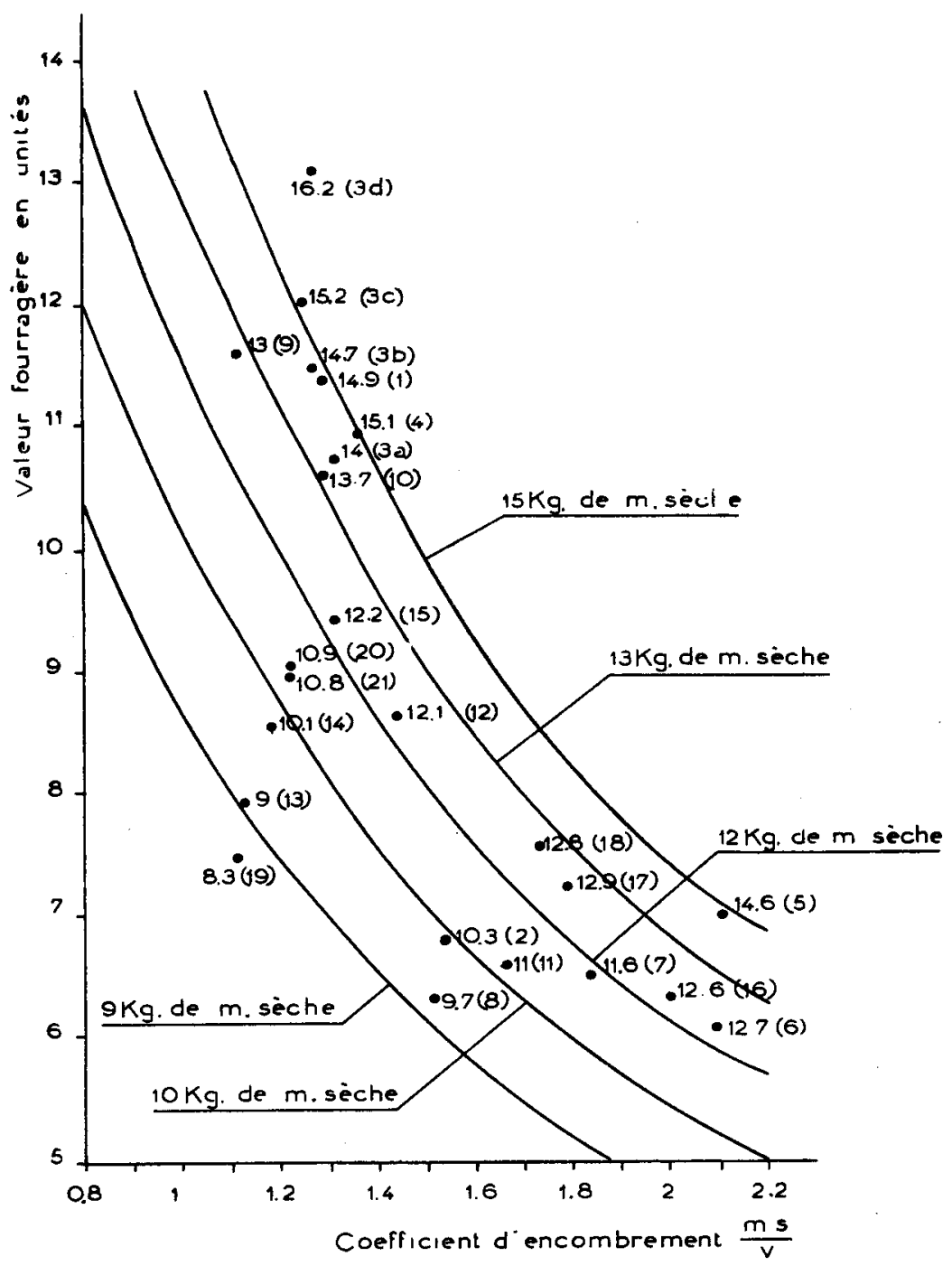

Graphigle i.

aux variations des valeurs fourragères en fonction de $\frac{\mathrm{MS}}{\mathrm{U}}$, qui sont parallèles entre elles, et donnent ainsi le système d'abaques du graphique 2 .

Reprenons maintenant les données de Fissmer contenues dans les tableaux $\mathrm{A} \mathrm{I}_{4}$ bis - A I4 ter - A I4 quater de la note VoISIN. Au moyen de la méthode 
décrite à l'annexe $I$, page 28 , de notre communication au Ve Congrès de Zootechnie, intitulée : "Normes pour l'alimentation énergétique ", il est aisé de calculer les valeurs fourragères des rations consommées par les sujets d'expérience, ainsi que les quantités de matière sèche correspondantes. Pour chaque

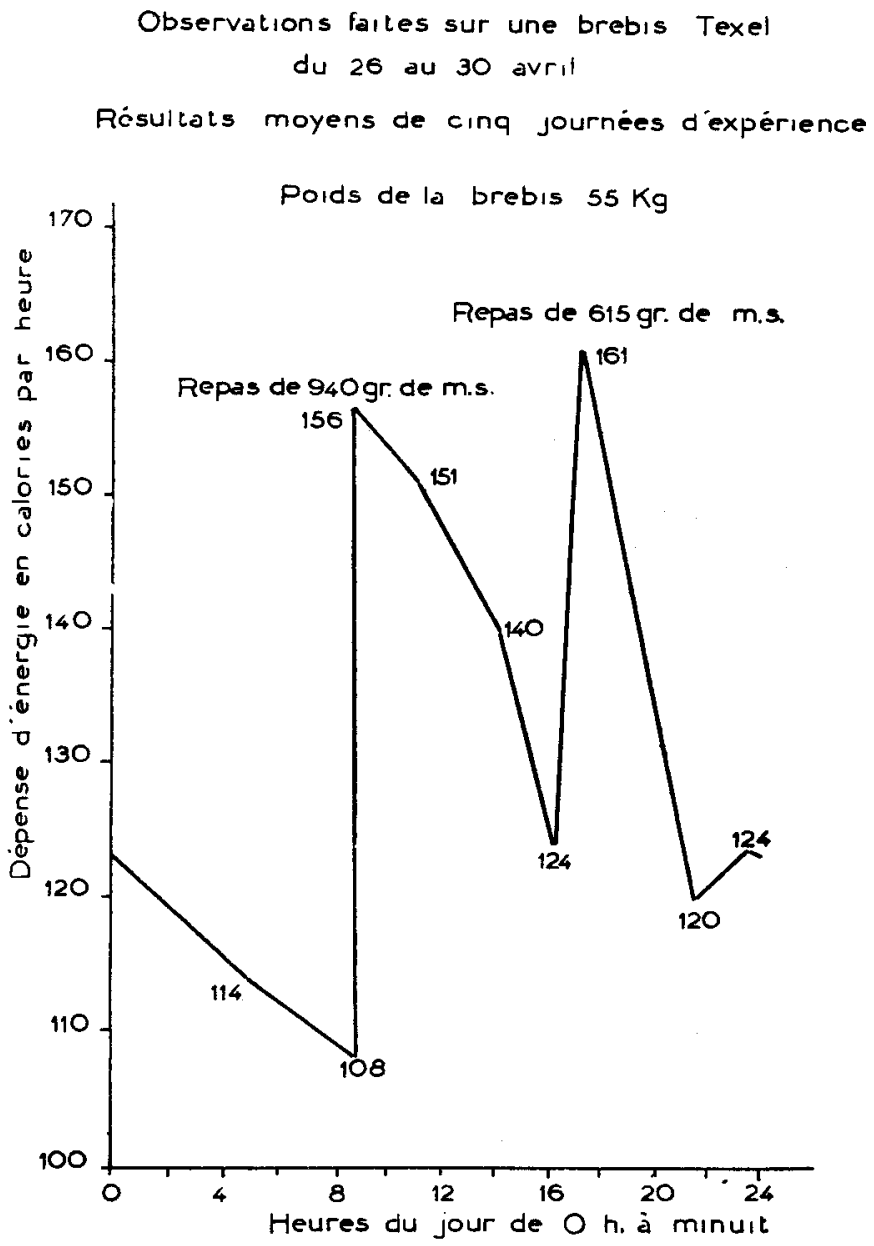

Graphinue 2 .

ration, nous obtenons ainsi un couple de données : "Valeur fourragère Coefficient d'encombrement ", que nous pouvons représenter par un point sur notre tableau.

Le graphique 2 montre la distribution remarquable de ces points, par rapport à la famille des courbes. Chaque point porte à côté de lui la quantité de matière sèche, exprimée en $\mathrm{kg}$ et un numéro de référence correspondant à la numération de la deuxième colonne à partir de la gauche du tableau $\mathrm{A}$ I4 quater. Nous remarquons que chaque point se place assez exactement à sa 
position théorique, par rapport à la courbe hypothétique correspondant à sa matière sèche. Ainsi, le point $3 c$ est très légèrement au-dessus de la courbe correspondant à $\mathbf{I} 5 \mathrm{~kg}$ de matière sèche, alors que la matière sèche consommée au cours de l'expérience $3 c$ est de $\mathrm{I}_{5}, 2 \mathrm{~kg}$. De même, les points $\mathrm{I}_{2}$ et $\mathrm{I}_{5}$, avec leurs teneurs en matière sèche respectives de $\mathrm{I} 2, \mathrm{I} \mathrm{kg}$ et $\mathrm{I} 2,2 \mathrm{~kg}$ sont au-dessus, mais assez près de la courbe des $\mathrm{I} 2 \mathrm{~kg}$ de $m . s$. La concordance avec la position théorique est d'autant plus frappante que les vaches n'avaient pas toutes exactement le même poids, et, par conséquent, la même ration d'entretien. Nous avons tendance à admettre, d'après cela, que c'est la " rapidité d'ingestion de la matière sèche " qui limite 1'absorption des aliments de base. Si les points $7,8, \mathrm{I} 3, \mathrm{I} 4, \mathrm{I} 9,20,2 \mathrm{I}$ correspondent à des faibles ingestions de matière sèche, malgré le faible coefficient d'encombrement des rations correspondantes, c'est que ces rations contenaient du Chou fourrager, aliment que les animaux ont ingéré lentement, sans doute parce qu'ils aiment moins la saveur de cet aliment que celle des autres, ainsi que Fissmer le dit lui-même. Des recherches systématiques sur les préférences des animaux pour les différentes nourritures auraient le plus grand intérêt, car, comme nous allons le voir, c'est de la plus ou moins grande rapidité de la consommation des aliments que dépendent principalement les pertes d'énergie métabolisable entraînées par l'alimentation.

Cette hypothèse est fortement étayée par les résultats d'expériences effectuées dans notre laboratoire, par la méthode de calorimétrie indirecte dite des " échanges gazeux ", sur des animaux appartenant à diverses espèces animales domestiques.

Le premier graphique (gràphique $n^{\circ} 2$ ci-joint) correspond aux dépenses horaires d'une brebis Texel, alimentée deux fois par jour, à 9 heures et à 77 heures, au moyen d'une masse alimentaire représentant I $555 \mathrm{~g}$ de matière sèche, divisée en deux repas. Nous avons porté en abscisses les heures de la journée et en ordonnées, les dépenses correspondantes en calories par heure.

Le deuxième indique, pour un intervalle moyen de 6 heures, la variation de la dépense d'une autre brebis qui a reçu des repas identiques à des intervalles de temps égaux, pendant plusieurs jours consécutifs, et qui a été systématiquement observée au bout de laps de temps plus ou moins éloignés du début du repas (graphique 3 ).

Le troisième se rapporte à des observations faites sur un porc de $50 \mathrm{~kg}$, recevant deux repas (graphique 4 ).

Enfin, le quatrième provient d'essais du même genre effectués sur des volailles d'un poids moyen de I $700 \mathrm{~g}$ alimentées 3 fois par jour (graphique no 5 ).

Tous ces documents montrent l'évolution du même phénomène : à la hausse de la dépense métabolique qui accompagne chaque repas, succède jusqu'au repas suivant une période de rémission. La chute de la dépense au cours de l'intervalle séparant un repas du suivant a une allure exponentielle, lorsqu'on l'étudie dans ses détails. Or, tandis que les monogastriques (porcs - volailles) 
peuvent prendre leurs repas en moins d'une demi-heure, les ruminants mettent plusieurs heures pour effectuer ce travail. On comprend ainsi pourquoi le rapport de l'énergie nette (utilisable pour les besoins du métabolisme basal et de la production) à l'énergie métabolisable (Énergie des ingesta diminutée de l'éner-

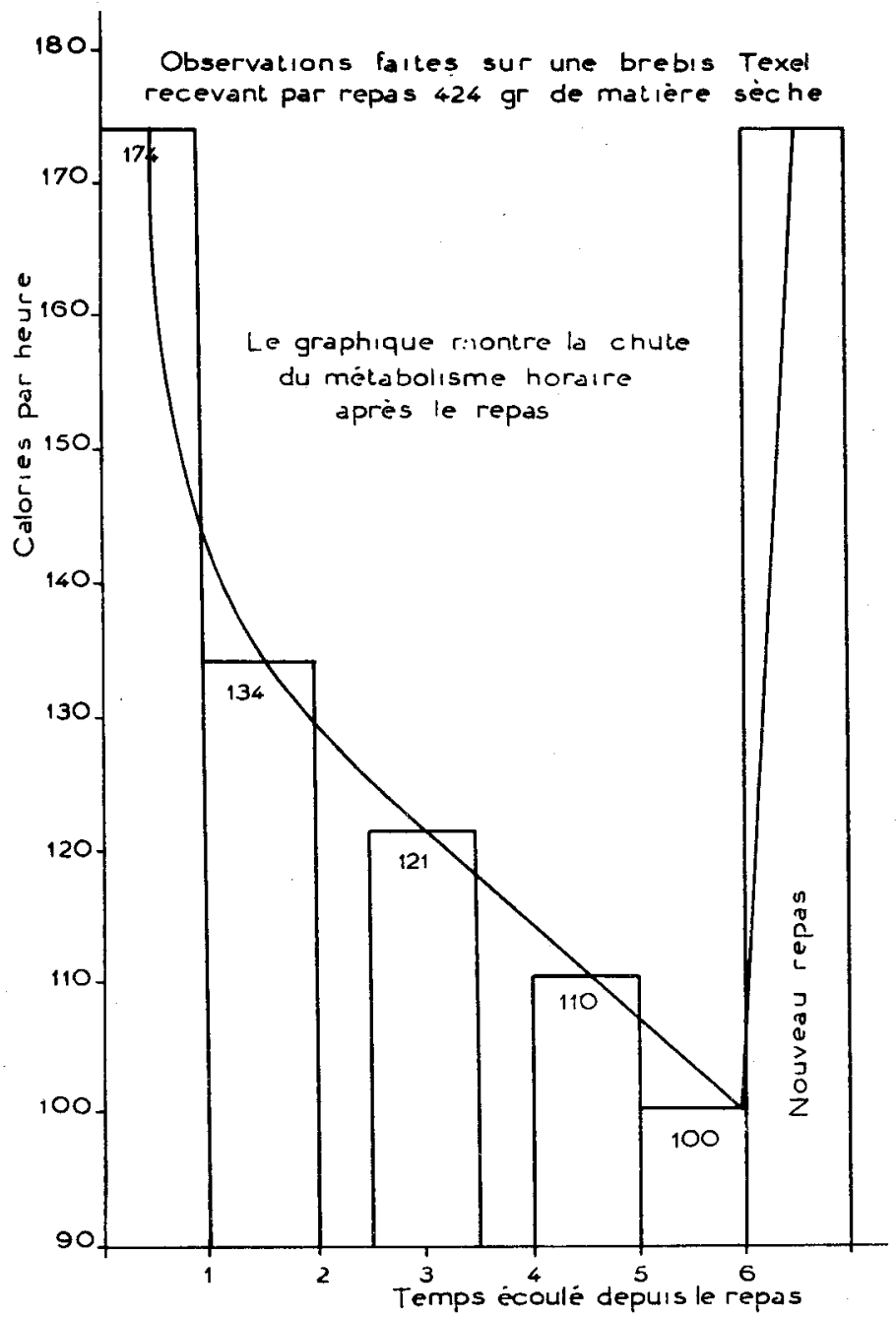

GRAPHIULE 3.

gie des excreta solides, liquides et gazeux) est beaucoup plus faible chez les bœufs et les moutons que chez les pores et les volailles. En diminuant la durée des repas, on peut augmenter le coefficient de transformation en tissus de croissance de la nourriture absorbée, comme l'ont nettement montré les expériences que nous avons nous-même effectuées sur des porcs et des volailles, avec la collaboration de M. FÉvrIER et de Mlle Geneviève LÉRY. 
L'observation attentive de ces documents permet de formuler les conclusoins suivantes :

I ${ }^{0}$ Pendant la durée entière d'un repas, la dépense énergétique de l'animal
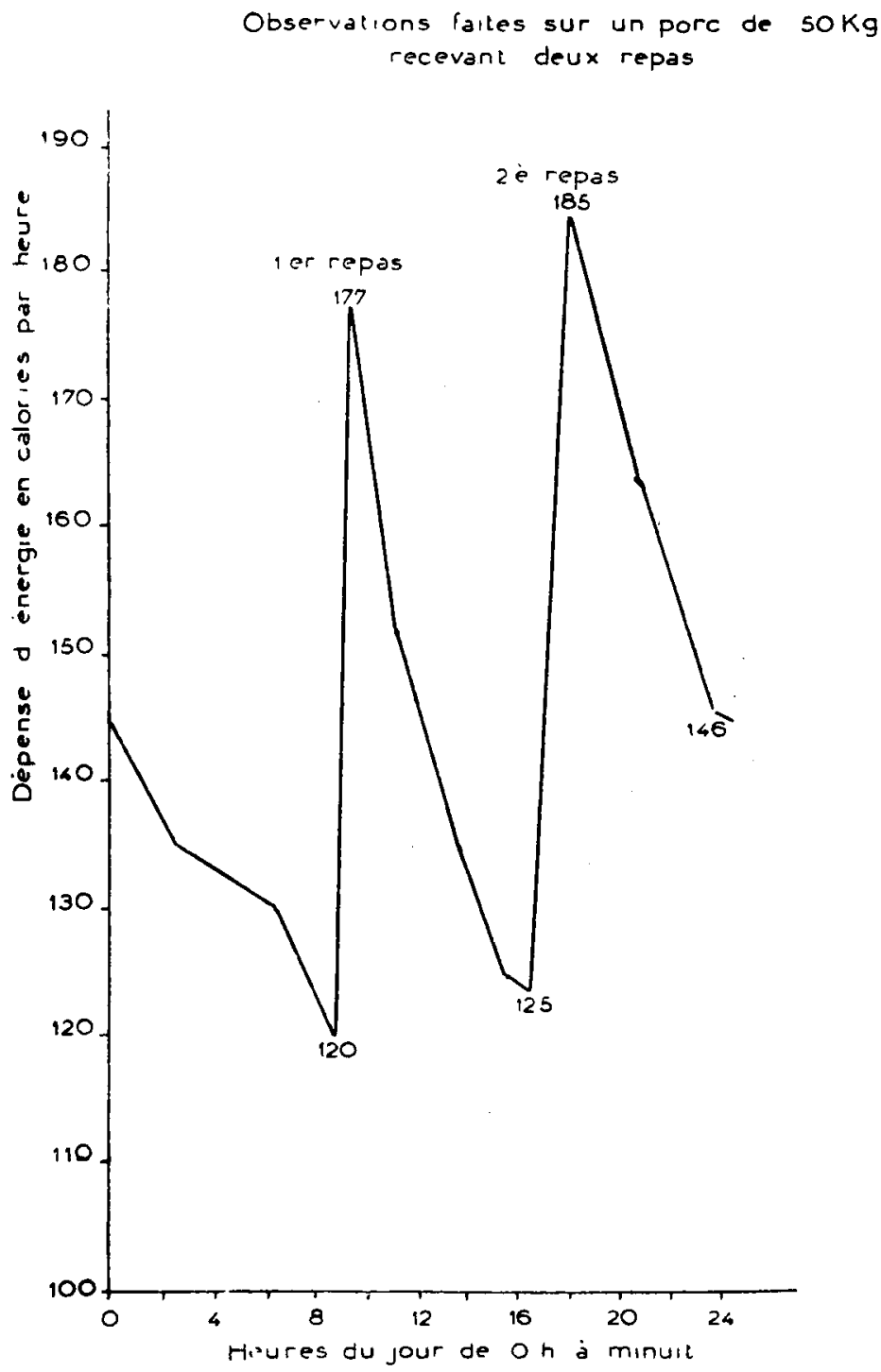

Graphinge 4.

passe par un maximum, puis décroît assez vite au cours de la phase de repos qui suit.

$2^{\circ}$ Lorsqu'on donne deux ou trois repas par jour, la pointe de métabolisme au cours d'un repas est nettement dépassée par celle du repas suivant, même 
si la quantité de matière sèche ingérée au cours de ce dernier est nẹttement audessous de celle du précédent (voir sur ce point le graphique 2 ).

$3^{\circ}$ Lorsque l'animal rumine, on ne perçoit pas pendant cet acte de hausse sensible de son métabolisme, car il n'y a pas de crochet anormal sur la courbe

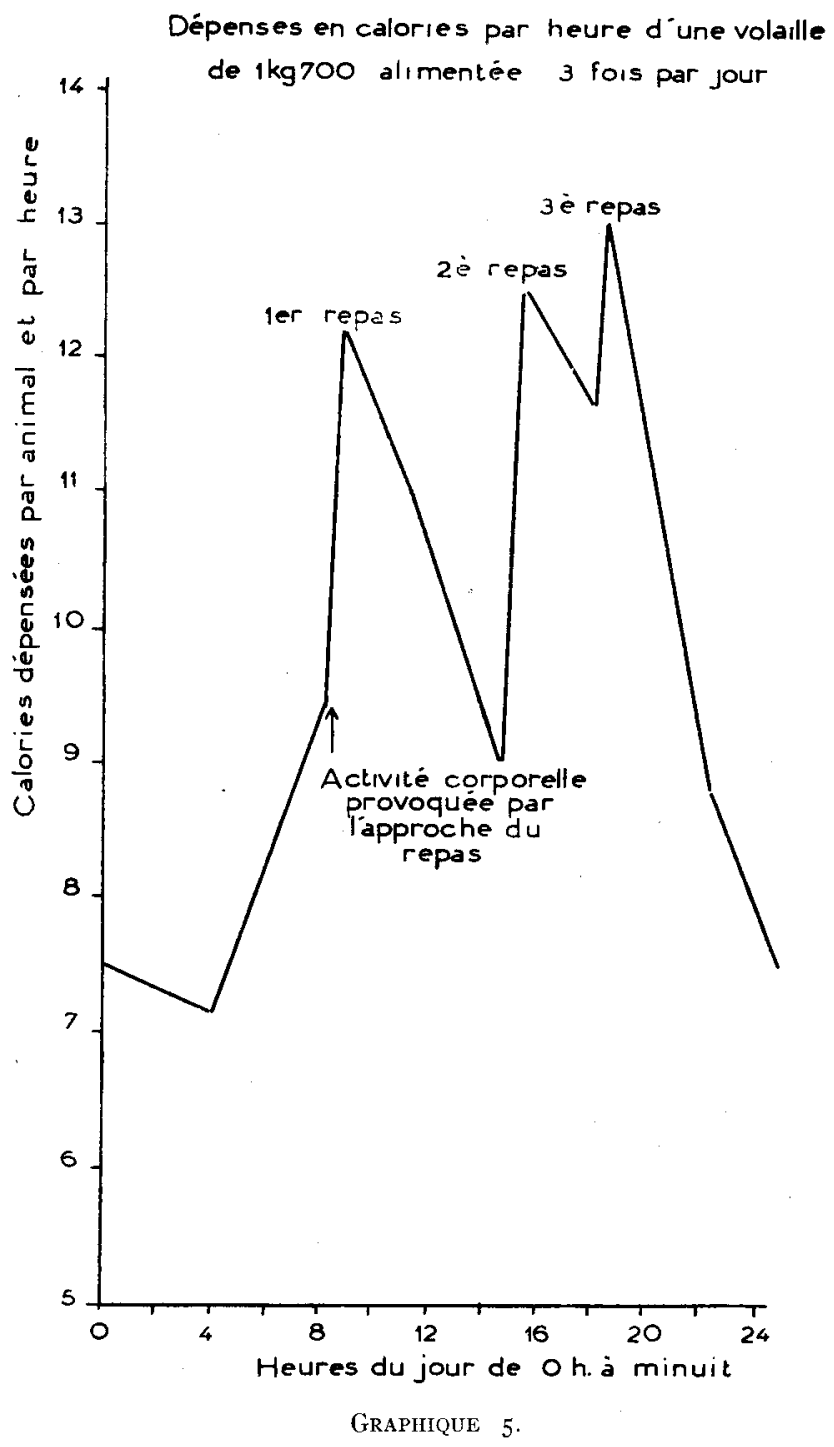

qui montre la chute de la dépense chez la brebis alimentée toutes les 6 heures (graphique 3). Or, au cours de cette expérience, l'animal a été observé pendant la rumination comme en l'absence de celle-ci.

La deuxième de nos conclusions infirme les calculs exposés en détail par M. Vorsin, dans la deuxième partie de son travail, car l'influence de la dépense 
énergétique pendant un repas sur les échanges métaboliques au cours du repas suivant n'est nullement proportionnelle à la masse de matière sèche consommée pendant ce deuxième repas, comme le présume la théorie basée sur l'existence d'une dépense uniforme d'énergie par coup de mâchoire.

Nous avons constaté nous-même que la différence entre la dépense énergétique totale par 24 heures d'un animal alimenté (correspondant à l'émission pendant le même temps de la chaleur latente et de la chaleur rayonnée) et la dépense correspondante d'entretien d'un animal à jeun, demeurant dans la position couchée 16 heures sur 24 , est très sensiblement voisine du résultat obtenu en multipliant la masse de matière sèche ingérée, exprimée en grammes, par I calorie (cf. le rapport de MM. DeLAGE et GASNIER. C. R. des travaux du Ve Congrès International de Zootechnie, Paris, 3, Io novembre I949).

D'après les observations de WALLACE et KeNNEDy (Un. Cornell Journal of $A g r$. Science, août 1944), une vache mange environ 5 heures sur 24, en donnant de 50 à 70 coups de gueule par minute, ce qui, à raison de $3 \mathrm{~g}$ environ par coup de mâchoire, correspond à l'ingestion de $55 \mathrm{~kg}$ d'herbe (à $22,5 \%$ de matière sèche), soit $\mathrm{I} 2,4 \mathrm{~kg}$ de matière sèche. La valeur énergétique d'une telle herbe, exprimée en énergie métabolisable, est de $2590 \mathrm{cal}$ par $\mathrm{kg}$ de matière sèche. Dans ces conditions, le bilan énergétique d'une vache de $600 \mathrm{~kg}$ est approximativement le suivant :

Besoin du métabolisme d'entretien. . . . . . . . . . . . . . . . . . . . . .

Supplément pour le déplacement corporel sur $4 \mathrm{kn}$, d'après S. BRovy ........

Dépense d'énergie supplémentaire provenant de la consommation des aliments, à rai-

son d'une calorie par gramme de matière seche ingérée $\ldots \ldots \ldots \ldots \ldots \ldots \ldots$

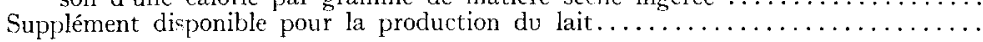

TotaI. correspondant à l'énergie metabolisable $: 2590 \times$ I $2,4 \ldots \ldots \ldots \ldots \ldots$

$8000 \mathrm{cal}$
I $200 \mathrm{cal}$.
I $2400 \mathrm{cal}$.
I0 5 I $6 \mathrm{cal}$.
$3 ?$ I 6 cal.

On sait que, par $\mathrm{kg}$ de lait à $4 \%$ de matière grasse la production d'énergie est de $75^{\circ}$ cal. La vache à l'herbage dans ces conditions peut donc produire I4 $\mathrm{kg}$ de lait sans faire appel à ses réserves, ce qui est parfaitement conforme aux résultats moyens observés par les divers expérimentateurs qui se sont occupés de l'alimentation à l'herbe. C'est donc bien la quantité de matière sèche qui domine le problème de la dépense métabolique chez l'animal alimenté. Mais il s'en faut évidemment que cette théorie trop simpliste puisse rendre compte dans tous leurs détails des phénomènes observés. La durée d'ingestion de cette matière sèche est le facteur de dépense fondamental, et il faut bien admettre que cette durée ne dépend pas seulement de la quantité de substance, mais encore de la constitution physique de celle-ci. Les aliments secs, qui ne se laissent pas insalivés facilement, sont plus longs à mâcher que les aliments humides, à quantités de matières sèches égales, et une correction devrait être apportée au calcul de la valeur fourragère, afin de tenir compte de ces faits.

Il reste donc encore beaucoup de travail pour les Chercheurs, et nous souhaitons ardemment que de jeunes expérimentateurs veuillent bien accepter de se pencher sur ces problèmes. En particulier, l'étude attentive des méca$\mathrm{n}^{\text {ismes }}$ physiologiques responsables de l'augmentation considérable du méta- 
bolisme énergétique pendant la consommation des aliments nous fournirait de précieux renseignements, dont le caractère d'utilité pratique est incontestable.

Déjà, nous savons qu'en raccourcissant dans toute la mesure du possible la durée des repas, nous pouvons diminuer de 7 à Io \% la dépense d'aliments ingérés par $\mathrm{kg}$ de viande, et il est bien certain que l'application d'une pareille méthode à l'alimentation du bétail laitier ne manquerait pas d'avoir sur l'économie de l'emploi des fourrages consommés la plus heureuse influence.

\section{BIBLIOGRAPHIE}

(I) Delage (J.) et Gasnier (A.). - I.'alimentation énergétique et azotée des animaux domestiques. Rapports particuliers. Ve Congrès International de Zootechnie. Paris, 3-10 novembre 1949.

(2) FÉvrier (R.) et Leroy (A. M.) - - Influence du mode de distribution des repas sur l'utilisation de la nourriture par le porc. Annales Agronomiques, n² 5, 1947 .

(3) FÉvrier (R.) et LEROY (A. M.). - Influence du nombre de repas sur l'utilisation de la nourriture par le porc. Annales Agronomiques, no 4 , i 949 .

(4) Leroy (A. M.). - Normes pour l'alimentation énergétique. Rapports généraux. Ve Congrès International de Zootechnie. Paris, 3-Io novembre 1949.

(5) LEROY (A. M.). - L'utilisation de l'énergie des aliments par les animaux. Alimentation et Agriculture. Bulletin Eurnpéen de la F.A.O. no 4, février-mars, 1948.

(6) LEROY (A. M.). - Influences du coefficient d'encombrement des rations pour vaches laitières. Bulletin technique d'Information. Ministère de l'Agriculture, Paris.

(7) LERoy (A. M.) et LÉRY (G.). - Étude de la durée de consommation des aliments par les volailles et l'influence de cette durée sur l'utilisation de l'énergie, apportée par la fraction digestible des matières ingérées. Annales de Zootechnie, no 1 , $195^{2}$.

(8) WALLACE et KENNEDY. - Grazing management practice. Journal of agricultural science, août, I944. Voir également dans la partie Documentation du présent fascicule les traductions suivantes:

Fissmer (F. E.). - Comment rassasier l'appétit d'une vache à lait. Zeitschrift für Tierernährung und Futtermittel kunde-Band $V$, I.941.

LehmanN (F.). - L'Alimentation animale d'après l'état de nos plus récentes connaissances. Tierzïcht. Zeittfragen, 1927 . S. 21 . 